\title{
Programas de residência multiprofissional em saúde mental e a terapia ocupacional ${ }^{1}$
}

\author{
Multi-professional residency programs in mental health and \\ occupational therapy
}

\author{
Aline Silva de Moura $^{\mathrm{a}}$ (D), Éllen Cristina Riccib (D), Sabrina Helena Ferigato ${ }^{\mathrm{a}}$ (iD \\ ${ }^{a}$ Universidade Federal de São Carlos - UFSCar, São Carlos, SP, Brasil.

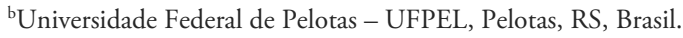

Como citar: Moura, A. S., Ricci, E. C., \& Ferigato, S. H. (2021). Programas de residência multiprofissional em saúde mental e a terapia ocupacional. Cadernos Brasileiros de Terapia Ocupacional, 29, e2951. https://doi.org/10.1590/2526-8910.ctoAO2235

\begin{abstract}
Resumo
Introdução: As Residências Multiprofissionais em Saúde Mental são formaçóes no/para o Sistema Único de Saúde e apresentam potencial transformador de práticas, em sintonia com a Reforma Psiquiátrica. Os terapeutas ocupacionais têm contribuição histórica no campo da saúde mental e há escassez de estudos que abordem sobre sua inserção nesta modalidade de formação. Objetivo: Realizar o mapeamento e análise da inserção da terapia ocupacional nos programas de residência multiprofissional em saúde mental no Brasil. Método: Estudo exploratório documental, com ênfase na análise descritiva de documentos institucionais, realizada em duas etapas: 1) busca ativa dos programas de residência multiprofissional em saúde mental e 2) mapeamento da terapia ocupacional nos programas de residência de saúde mental. Resultados: A terapia ocupacional está presente em 31 programas de residência multiprofissional em saúde mental no país, distribuídos em doze estados, com 83 vagas ofertadas no ano de 2020 e 75 no ano de 2021, sendo a quarta profissão com maior oferta de vagas dentre as dez identificadas. Embora haja uma insuficiência de vagas em comparação a outras profissões, o campo da saúde mental representa a maior oferta de vagas em residências para terapeutas ocupacionais. Conclusão: A formação interprofissional pode favorecer uma ampliação de outros profissionais acerca da terapia ocupacional e há a necessidade de maiores estudos para compreender de que modo esses processos formativos acontecem na prática.
\end{abstract}

Palavras-chave: Formação Profissional, Saúde Mental, Terapia Ocupacional, Educação Interprofissional.

\footnotetext{
${ }^{1}$ Parte deste estudo está vinculado à Pesquisa de Doutorado em andamento da primeira autora, sob orientação da última, intitulada "A prática de preceptoria em programas de residência multiprofissional em saúde mental", junto ao Programa de PósGraduação em Terapia Ocupacional da Universidade Federal de São Carlos.
} 


\begin{abstract}
$\underline{\text { Abstract }}$
Introduction: Multi-professional residencies in mental health are training courses in/for the Sistema Único de Saúde and have the potential to transform practices, in line with the Psychiatric Reform. Occupational therapists have a historical contribution in the field of mental health and there is a scarcity of studies addressing their insertion in this type of training. Objective: To map and analyze the insertion of occupational therapy in multi-professional residency programs in mental health in Brazil. Method: Exploratory documentary study, with emphasis on the descriptive analysis of institutional documents, carried out in two stages: 1) active search for multidisciplinary mental health residency programs and 2) mapping of occupational therapy in mental health residency programs. Results: Occupational therapy is present in 31 multi-professional residency programs in mental health in the country, distributed in twelve states, with 83 places offered in 2020 and 75 in 2021, being the fourth profession with the highest offer of places among the ten identified. Although there is an insufficiency of vacancies compared to other professions, the field of mental health represents the largest offer of vacancies in residences for occupational therapists. Conclusion: Interprofessional training can favor an expansion of other professionals about occupational therapy and there is a need for further studies to understand how these training processes happen in practice.
\end{abstract}

Keywords: Professional Training, Mental Health, Occupational Therapy, Interprofessional Education.

\title{
Introduçáo
}

A Residência em Área Profissional de Saúde, modalidade uniprofissional e multiprofissional, é uma pós-graduação caracterizada pela articulação entre ensinoserviço-comunidade, a qual favorece a inserção qualificada de profissionais de saúde no/para o Sistema Único de Saúde (SUS). Regulamentada em 2005, por meio da Lei $\mathrm{n}^{\circ} 11.129$, pode abranger as seguintes categorias profissionais: Biomedicina, Ciências Biológicas, Educação Física, Enfermagem, Farmácia, Fisioterapia, Fonoaudiologia, Medicina Veterinária, Nutrição, Odontologia, Psicologia, Serviço Social e Terapia Ocupacional, além de Física Médica e Saúde Coletiva, incluídas posteriormente (Brasil, 2006, 2014).

As Residências Multiprofissionais em Saúde (RMS) se apresentam como espaços privilegiados de formação, tendo como questóes centrais a integralidade e a interprofissionalidade (Silva, 2018) em diferentes campos da saúde. Para Garcia Junior \& Yasui (2018), seus processos formativos buscam um campo comum de práticas e experiências, embora a exclusão da categoria médica na composição desses programas ainda revele um modo hegemônico de se pensar a saúde.

A necessidade de transformação nos processos de formação para o trabalho é abordada por Ceccim \& Feuerwerker (2004), que consideram que o trabalho em saúde possui uma característica que abrange a atençáo à saúde, a gestão, o ensino e o controle social, compondo o que denominam por quadrilátero da formação em saúde. O ensino, 
porém, é um dos pontos menos problematizados, e tanto gestão quanto instituições formadoras ainda mantêm modelos de formação essencialmente conservadores.

$\mathrm{O}$ modo de formação e intervenção em saúde proposto pelas residências multiprofissionais tem a possibilidade de "[...] afetar o cotidiano dos serviços e, ao mesmo tempo, afetar a Universidade com a realidade dos serviços [...]"; com isso, favorecem a integração ensino-serviço (Garcia Junior \& Yasui, 2018, p. 162). Destacamos que, nos programas de residência, outras instituiçóes formadoras e executoras podem estar vinculadas, sendo que a Universidade não compóe a sua totalidade.

Uma das modalidades de Residência Multiprofissional é aquela que caracteriza o campo da Saúde Mental. As residências em saúde mental apresentam potencial transformador de práticas, em sintonia com a Reforma Psiquiátrica (RP), possibilitando um aprendizado que extrapola o campo do conhecimento teórico e prático, incluindo atitudes, posicionamentos políticos, habilidades e competências para lidar com as especificidades do cuidado em saúde mental, tendo a subjetividade como sua matéria prima (Coelho et al., 2017). Desse modo, é um processo de formação profissional que investe na construção de sentidos para as experiências dos estudantes em campo (Emerick \& Onocko-Campos, 2016) e para o fortalecimento do próprio campo da saúde mental nas universidades e na rede pública de saúde.

Caracterizada por uma ruptura em relação ao conceito de loucura e do saber psiquiátrico, a RP coloca o modo asilar em cheque e passa a considerar o sofrimento humano em sua complexidade, não apenas como objeto simples da doença mental (Yasui, 2010). É um processo social complexo, que entrelaça diferentes dimensôes: técnico-assistencial, teórico-conceitual, jurídico-política e sociocultural (Amarante, 2007).

Emerick \& Onocko-Campos (2016), ao considerarem a complexidade da Reforma Psiquiátrica Brasileira, a qual requer uma transformação do paradigma de cuidado, apontam para a demanda de novos processos de formação

Para novos caminhos, fazem-se necessárias novas perguntas. Para aprender a lidar com novos modos de cuidado, com a complexidade que se apresenta na vida em território a céu aberto, novos percursos de formação devem ser trilhados [...]. Se buscamos transformar os modos como as pessoas são tratadas para transformar seus sofrimentos, havemos de investir na transformação dos modos pelos quais formamos profissionais para atuarem no processo de Desinstitucionalização (Emerick \& Onocko-Campos, 2016, p. 99).

O compromisso com a Reforma Psiquiátrica e com o Movimento da Luta Antimanicomial marca a história da terapia ocupacional (TO). Nesse sentido, preconiza-se a formação de profissionais implicados com a inclusão social e emancipação de sujeitos individuais e coletivos (Lussi et al., 2019).

Diferentes estudos abordam a formação em saúde mental na terapia ocupacional no âmbito da graduação (Palm et al., 2015; Lins \& Matsukura, 2015a, 2015b). Ao discutir sobre essa formação a partir da perspectiva de docentes vinculados à cursos de graduação em TO, o estudo de Lins \& Matsukura (2015a) revelou como desafios a distância entre o que é preconizado nas políticas públicas e o observado na prática, assim como a 
limitação das grades curriculares. Quanto à formação em saúde mental pós-graduada em TO, em especial no âmbito das residências, há uma escassez de estudos.

Desse modo, ao considerarmos a importante contribuição dos terapeutas ocupacionais no cuidado em saúde mental e a potência das residências multiprofissionais para uma formaçáo qualificada nesse cenário, torna-se relevante conhecer de que modo a profissão tem participado e se inserido nesses programas.

Nesse sentido, tomaremos como objeto de estudo do presente artigo a inserção da terapia ocupacional em programas de residência em saúde mental. Nosso objetivo foi realizar o mapeamento e análise da inserção da terapia ocupacional nos programas de residência multiprofissional em saúde mental no Brasil.

\section{Metodologia}

Partimos de um estudo exploratório documental, com ênfase na análise de documentos institucionais, e de reflexôes das autoras fundamentadas na atenção psicossocial e educação interprofissional para o mapeamento e análise da inserção da terapia ocupacional nos programas de residência multiprofissional em saúde mental no Brasil.

A pesquisa documental é aquela que utiliza materiais que não receberam um tratamento analítico (Gil, 2018) e que permite ampliar o conhecimento a respeito de um determinado fenômeno, o qual necessite de contextualização histórica e sociocultural (Sá-Silva et al., 2009).

Os dados foram coletados entre outubro/2020 e janeiro/2021, com base nos seguintes documentos: editais dos processos seletivos dos programas de residência em saúde mental; sites das instituições executoras e formadoras dos programas; e projetos políticos pedagógicos dos programas. Esse processo se deu em duas etapas:

Etapa 1: Busca ativa dos programas de residência multiprofissional em saúde mental

Solicitamos à equipe técnica da Residência Multiprofissional do Ministério da Educação (MEC), em outubro de 2020, uma relação dos programas de residência em Saúde Mental, o que resultou em uma lista com 77 programas.

A partir dessa ação, realizamos busca manual dos editais dos processos de seleção para ingresso em 2020 e 2021 disponíveis na internet, através da plataforma de buscas Google e nos sites das instituiçôes.

Como critérios de inclusão, nessa etapa tivemos: programas caracterizados como multiprofissionais e que estivessem ativos (com pelo menos uma turma de residentes em curso em 2020 e/ou 2021), contendo a palavra "saúde mental", "atenção psicossocial" ou "atenção ao usuário de álcool e/ou outras drogas" no seu título ou em sua área de concentração.

Etapa 2: Mapeamento da inserção da terapia ocupacional nos programas de residência em saúde mental

Após a filtragem inicial dos programas de residência multiprofissional em saúde mental, utilizou-se como critério de inclusão os que ofertaram vagas para terapia ocupacional. 
Em seguida ao mapeamento das etapas 1 e 2, utilizou-se um banco informatizado por meio do programa Excel , visando ao tratamento e organização dos dados, para análise descritiva.

Foi realizada sistematização numérica dos programas de saúde mental, sua distribuição por região geográfica, distribuição de vagas por categoria profissional, com ênfase na inserção da terapia ocupacional.

Além da análise da inserção, foram identificadas algumas especificidades quanto à organização curricular dos programas e especificidades do núcleo profissional destacadas nos projetos político pedagógicos e nos editais de seleção.

Ao fim do processo, os resultados foram categorizados em 2 eixos: (1) Distribuição regional e oferta de vagas nos programas de Residência Multiprofissional em Saúde Mental; (2) Terapia Ocupacional nos programas de residência em Saúde Mental.

\section{Resultados e Discussáo}

$\mathrm{Na}$ primeira etapa, a partir da lista inicial de 77 programas, excluímos os uniprofissionais (5), aqueles que estavam duplicados (10), os que não possuíam dados suficientes para localização do programa (2), os inativos (11) ou que não foi possível localizar editais (10).

Durante a busca, incluímos 13 programas não listados no documento oficial enviado pelo MEC, os quais foram identificados em editais integrados com outros programas e/ou em busca manual pela internet.

No total, foram identificados 52 programas de residência em saúde mental, em 43 editais, dos quais foram coletados dados referentes ao número total de programas de residência em saúde mental com oferta de vagas para ingresso nos anos 2020 e 2021, sua distribuição geográfica e oferta de vagas total e por categoria profissional.

$\mathrm{Na}$ segunda etapa, a busca por residências que ofertavam vagas para a terapia ocupacional resultou em 31 programas, publicados em 26 editais. Nesses editais, além dos dados referidos na etapa anterior, buscou-se também dados quanto ao objetivo e/ou missão dos programas e dados que trouxessem algo da especificidade da terapia ocupacional; no caso, apenas a indicação de referência bibliográfica específica para a categoria profissional (localizado em 13 editais).

Nos sites institucionais, buscou-se o objetivo do programa e/ou sua missão, quando este não esteve explícito nos editais. Buscamos ainda os projetos políticos pedagógicos desses programas, sendo localizados apenas 3, nos quais se buscou dados referentes ao currículo adotado pelo programa e especificidades da terapia ocupacional abordadas no documento.

Sendo assim, entre 2020 e 2021, contabilizou-se 52 programas de residência em saúde mental e 31 deles com vagas para terapia ocupacional. Segue abaixo a descrição e discussão dos achados. 
Distribuição regional e oferta de vagas nos programas de Residência Multiprofissional em Saúde Mental

Os 52 programas de residência em saúde mental que identificamos estáo distribuídos nas cinco regiōes do país, em 19 estados, com uma oferta total de 525 vagas (com ingresso em 2020) e 492 vagas (com ingresso em 2021), de acordo com a Tabela 1.

Tabela 1. Distribuição de vagas e de programas de Residência Multiprofissional em Saúde Mental, com ingresso nos anos 2020 e 2021, por regióes e estados brasileiros.

\begin{tabular}{|c|c|c|c|c|c|c|}
\hline Regiáo/Estados & No Programas & $\%$ & Vagas 2020 & $\%$ & Vagas 2021 & $\%$ \\
\hline Norte & 4 & 7,7 & 40 & 7,6 & 42 & 8,5 \\
\hline Amapá & 1 & 1,9 & 7 & 1,3 & 7 & 0 \\
\hline Pará & 1 & 1,9 & 13 & 2,5 & 13 & 2,8 \\
\hline Rondônia & 1 & 1,9 & 6 & 1,1 & 6 & 1,3 \\
\hline Tocantins & 1 & 1,9 & 14 & 2,7 & 16 & 3,4 \\
\hline Nordeste & 13 & 25 & 150 & 28,6 & 141 & 28,6 \\
\hline Bahia & 2 & 3,8 & 13 & 2,5 & 14 & 3,0 \\
\hline Ceará & 3 & 5,8 & 68 & 13,0 & 68 & 14,6 \\
\hline Paraíba & 1 & 1,9 & 20 & 3,8 & 20 & 4,3 \\
\hline Pernambuco & 5 & 9,6 & 36 & 5,9 & 36 & 7,7 \\
\hline Rio Grande do Norte & 1 & 1,9 & 3 & 0,6 & 3 & 0,6 \\
\hline Sergipe & 1 & 1,9 & 10 & 1,9 & 0 & 0 \\
\hline Centro-Oeste & 4 & 7,7 & 46 & 8,8 & 51 & 10,4 \\
\hline Distrito Federal & 3 & 5,8 & 43 & 8,3 & 48 & 10,3 \\
\hline Mato Grosso do Sul & 1 & 1,9 & 3 & 0,6 & 3 & 0,6 \\
\hline Sudeste & 17 & 32,7 & 173 & 32,9 & 151 & 30,7 \\
\hline Espírito Santo & 1 & 1,9 & 20 & 3,8 & 20 & 4,3 \\
\hline Minas Gerais & 4 & 7,7 & 32 & 6,1 & 27 & 5,8 \\
\hline Rio de Janeiro & 3 & 5,8 & 46 & 8,8 & 46 & 5,6 \\
\hline São Paulo & 9 & 17,3 & 75 & 14,4 & 58 & 12,5 \\
\hline Sul & 14 & 26,9 & 117 & 22,3 & 108 & 21,9 \\
\hline Paraná & 3 & 5,8 & 19 & 3,6 & 18 & 3,9 \\
\hline Santa Catarina & 1 & 1,9 & 15 & 2,9 & 15 & 3,2 \\
\hline Rio Grande do Sul & 10 & 19,2 & 83 & 15,9 & 75 & 16,1 \\
\hline Total & 52 & 100 & 525 & 100 & 492 & 100 \\
\hline
\end{tabular}

Fonte: Elaboração própria (2021).

Observamos que há um maior número de programas de residência em saúde mental na região Sudeste (17).

O estudo de Sarmento et al. (2017), que analisou a distribuição da oferta de programas de residência multiprofissional em saúde no período de 2009 a 2015, observou um aumento dos programas ofertados no cenário nacional; porém, manteve-se 
a tendência de concentração na região Sudeste, em especial em São Paulo, seguida pelas regiôes Nordeste e Sul. As regiōes Norte e Centro-Oeste também foram as com menor número de oferta de programas.

Percebemos que, com relação ao número de vagas ofertadas por ano, a região Sudeste continua a apresentar o maior número, seguida da regiáo Nordeste.

Identificamos que, dos 52 programas de residência em saúde mental, 31 deles são ofertados nas capitais brasileiras e 21 estão em municípios do interior do Brasil. Dentre esses programas, 3 são regionais (com ofertas de vagas para diferentes municípios em uma única residência multiprofissional), sendo eles localizados nos seguintes estados: Espírito Santo (com vagas na capital e em outros três municípios), Ceará (com vagas na capital e em outros 11 municípios) e Rio Grande do Sul (com vagas em 4 municípios do interior do estado). Além disso, um dos programas se caracteriza-se como interestadual, incluindo nos cenários de prática municípios da macrorregião norte da Bahia e macrorregião de Petrolina, no estado de Pernambuco.

Não localizamos programas de residência em Saúde Mental ativos nos seguintes estados: Acre, Alagoas, Amazonas, Maranhão, Mato Grosso, Piauí e Roraima. Além disso, nos estados de Santa Catarina e Rondônia, há oferta de programas apenas no interior.

Os programas de Residência Multiprofissional em Saúde apresentam como eixos norteadores a regionalização, a descentralização e a interiorização do trabalho em saúde. Desse modo, são uma "[...] proposta de formação com potencial para fixação de profissionais em regiōes onde há restrição de oferta de serviços de saúde, fortalecendo os laços de responsabilidade com as comunidades" (Sarmento et al., 2017, p. 421). Observamos que esse processo de interiorização está presente em nove estados (que concentram, como já citado, os 21 municípios com programas de residência em saúde mental). Identificamos, porém, que há a necessidade de estudos para identificação de ociosidade de vagas e se essas ofertas têm favorecido a fixação de profissionais, preconizadas pela RMS.

Onocko-Campos et al. (2019), a partir de dados informados pelo Ministério da Saúde ${ }^{2}$, apontam que, até o ano de 2010, existiam apenas seis vagas para residência multiprofissional em saúde mental, distribuídas em um único estado. Referem que houve expressivo aumento de ofertas nos anos de 2013 (95 vagas), 2014 (68 vagas), 2015 (54 vagas) e, em 2017, eram 29 programas, distribuídos em dez estados brasileiros, com 300 vagas.

Observamos, assim, uma expansão dos programas de residência em saúde mental nos últimos anos, até 2020. Em 2021, tivemos uma queda na oferta de 33 vagas. A Figura 1 retrata essa evolução do número de vagas por ano.

\footnotetext{
${ }^{2}$ Dados informados pelo Ministério da Saúde, via e-SIC (No 2368225), em novembro de 2017. Nota Técnica No 42- SEI/2017 - CGEAP/DEPREPS/SGTES/MS (Onocko-Campos et al., 2019).
} 


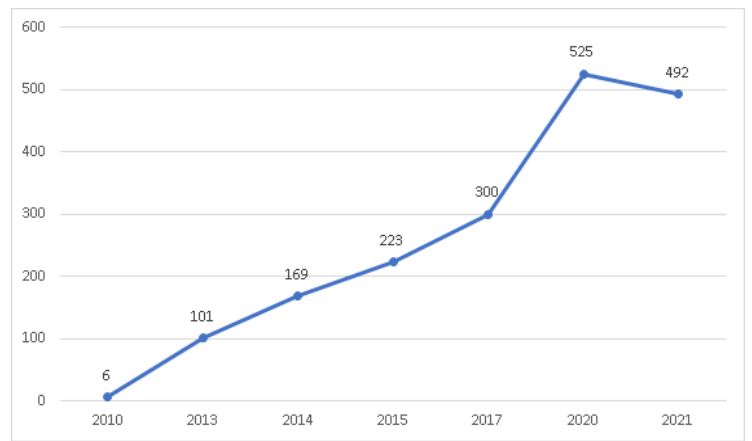

Figura 1. Evolução do número de vagas ofertadas por ano nos programas de Residência Multiprofissional em Saúde Mental (2010-2021). Fonte: Elaboração própria (2021).

\section{Categorias profissionais que compöem os programas de residência multiprofissional em saúde mental}

Para ser caracterizado como um programa de residência multiprofissional, devem estar presentes ao menos três categorias profissionais distintas. Em nosso estudo, identificamos dez categorias profissionais nos programas de residência em saúde mental: enfermagem, educação física, farmácia, fisioterapia, fonoaudiologia, nutrição, odontologia, psicologia, serviço social e terapia ocupacional. A Figura 2 apresenta a distribuição de vagas por profissão nos anos de ingresso de 2020 e 2021.

Os programas de residência multiprofissional em saúde mental ofertaram vagas para no mínimo três categorias profissionais e no máximo sete. $\mathrm{O}$ mais prevalente, identificado em dezoito programas, foi a composição de apenas três categorias profissionais, sendo a psicologia, a enfermagem e o serviço social (respectivamente). Esta composição esteve presente em treze programas de residência.

Quinze programas de residência contavam com 5 categorias profissionais, sendo que, em sete delas, estiveram presentes as três profissóes anteriores, a terapia ocupacional e a educação física (respectivamente, o quarto e quinto núcleo profissional com maior oferta de vagas).

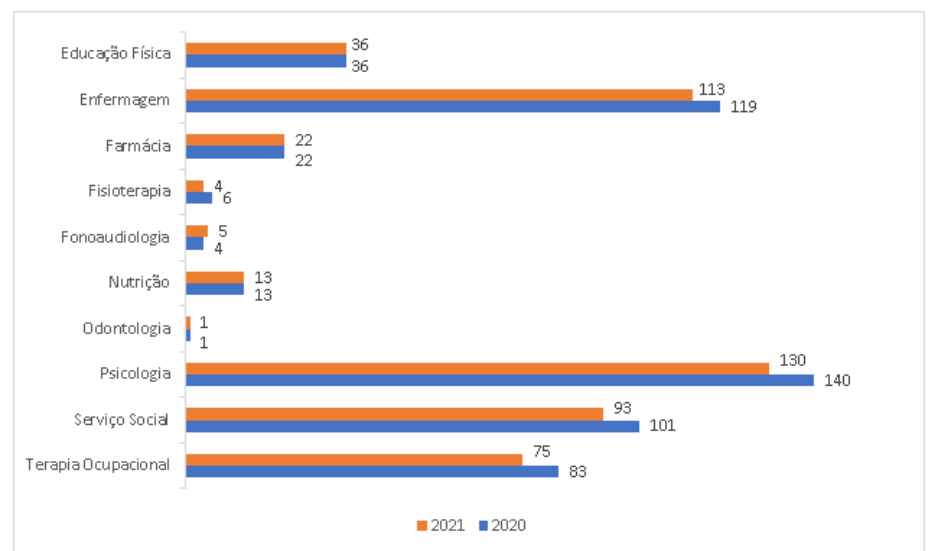

Figura 2. Distribuição quantitativa de vagas nos programas de Residência Multiprofissional em Saúde Mental, por categoria profissional, com ingresso nos anos de 2020 e 2021. Fonte: Elaboração própria (2021). 
A Organização Mundial da Saúde (2010, p. 7) define a educação interprofissional como "[...] o aprendizado que ocorre quando estudantes de duas ou mais profissóes aprendem sobre os outros, com os outros e entre si para possibilitar a colaboraçáo eficaz e melhorar os resultados de saúde", os quais são favorecidos nos programas de RMS.

A constituição de equipes interdisciplinares e interprofissionais tem como pressupostos: superar a fragmentação do trabalho e da individualização biomédica; buscar a reconstituição da integralidade do trabalho coletivo em saúde; e qualificar os profissionais, visando democratizar o contexto do trabalho e a efetivação de um cuidado integral (Peduzzi et al., 2020).

A finalidade da educação interprofissional está na direção do desenvolvimento do trabalho interprofissional e de práticas colaborativas. Entretanto, ter diferentes composiçôes com as dez categorias profissionais identificadas nos programas de residência multiprofissional em saúde mental não garante que ocorra um trabalho na lógica interprofissional, preconizado pelas RMS.

\section{A terapia ocupacional nos Programas de Residência Multiprofissional em Saúde Mental}

A terapia ocupacional atualmente compóe 31 programas de Residência em Saúde Mental, distribuídos em doze estados brasileiros. De acordo com os editais dos processos seletivos identificados, foram ofertadas 83 vagas para terapeutas ocupacionais para ingresso no ano de 2020 e 75 vagas para ingresso em 2021. Isso significa dizer que, no total das 33 vagas multiprofissionais reduzidas no último ano, 24,2\% da perda das vagas impactaram diretamente a inclusão de terapeutas ocupacionais em programas de residência em saúde mental.

Das 31 residências em saúde mental que contam com terapeutas ocupacionais, não houve abertura de vagas em dois programas, localizados no estado de São Paulo (nos municípios de Sorocaba e Santos).

Além disso, houve redução de vagas para a terapia ocupacional em dois programas de residência: um no Rio Grande do Sul, que é regional e que teve redução geral de vagas (de 17 para 8), sendo que, para a terapia ocupacional, o número de vagas passou de 3 para 1; e o outro no Ceará, o qual, apesar de ter mantido o número geral de vagas (53), reduziu 4 vagas ofertadas para terapia ocupacional (que passou de 11 para 7). Observamos nesse último programa que essas vagas foram realocadas para o curso de educação física.

Houve aumento de vagas em nossa profissáo em apenas um programa, localizado no Distrito Federal, no qual a terapia ocupacional passou de 2 para 5 vagas, sendo que, no quadro geral, houve aumento de apenas uma vaga, assim como redução de duas vagas para a enfermagem (de 6 para 4).

Não tivemos como objetivo, neste momento, mapear os motivos de redução de vagas para a terapia ocupacional e a náo abertura de novas turmas dos programas. Acreditamos que o cenário de pandemia da COVID-19 pelo qual estamos vivendo tenha impactado o desenvolvimento das residências, assim como um contexto de sucateamento de serviços públicos; porém, há a necessidade de investigação.

Dos 31 programas de residência com ofertas de vagas para a terapia ocupacional, dois são de Saúde Mental Coletiva; três estão voltados para a atenção ao usuário de álcool e 
outras drogas (porém, com diferentes terminologias no nome do programa, tal como "dependência química" e "atenção integral ao usuário de drogas"); um está voltado exclusivamente para a saúde mental de adultos; um para a infância e juventude; um se caracteriza como programa de residência hospitalar, mas com área de concentração na saúde mental; e outro com ênfase na atenção básica. $O$ restante (22) não traz especificaçóes nesse sentido. Porém, traz diferenças nos termos adotados nos nomes de seus programas: Rede de Atenção Psicossocial (2); Saúde Mental no Sistema Público de Saúde (1); e Saúde Mental (19).

O termo "Residência Integrada" caracteriza o nome de sete desses programas de RMS em saúde mental. De acordo com Torres et al. (2019), o termo ainda não foi adotado oficialmente pela Comissão Nacional de Residências Multiprofissionais em Saúde, embora seja encontrado em alguns programas e represente um modelo de se pensar e fazer as residências.

Para Poellnitz \& Silva (2019), os termos são expressôes que nomeiam fenômenos e/ou objetos e podem ter conceitos atrelados a eles. O que essas diferentes denominaçóes dos programas de residência dizem dos modelos de atençáo em saúde mental?

A partir dos editais e sites institucionais, buscamos identificar os objetivos dos programas de residência em saúde mental nos quais temos a inserção da terapia ocupacional. Percebemos que, em geral, há um alinhamento com as diretrizes do SUS e da Reforma Psiquiátrica em suas apresentações.

Percebemos que houve grande referência à Rede de Atenção Psicossocial. Criada em 2011 pela portaria 3088, tem como um de seus objetivos a garantia da "[...] articulação e integração dos pontos de atenção das redes de saúde no território, qualificando o cuidado por meio do acolhimento, do acompanhamento contínuo e da atenção às urgências" (Brasil, 2011, p. 2). Ainda de acordo com sua portaria, objetiva garantir o acesso da população à atenção psicossocial e, em especial, supóe a construção de espaços de convívio e sustentação das diferenças na comunidade. Para tanto, compóem essa rede: Unidades Básicas de Saúde; Centros de Convivência; Centros de Atenção Psicossocial; serviços de urgência e emergência; Unidades de Pronto Atendimento 24 horas; Serviços de Atenção em Regime Residencial; enfermarias especializadas em Hospital Geral; entre outros serviços (Brasil, 2011).

Os diferentes serviços foram identificados como cenários de prática dos programas de residência; em especial, a atenção básica e as diferentes modalidades de Centros de Atenção Psicossocial. Nesse sentido, reforçamos a importância dos programas de residência para a transformação de práticas em saúde mental, superando o modo asilar e dando lugar para uma assistência em rede, comunitária e interdisciplinar.

Identificamos que alguns programas referem possuir como cenário de prática "Unidades de Internação" e "Instituto de Psiquiatria". Não foi possível, porém, identificarmos a que modelo de atenção tais instituiçôes se referem (e se estão, ou não, vinculados a enfermarias especializadas em hospital geral, conforme inicialmente consta na composição da Rede de Atenção Psicossocial, ou se referem a hospitais psiquiátricos). Salientamos que nossas reflexóes partem da leitura de documentos institucionais, não sendo possível avaliar de que modo acontecem suas práticas.

Amarante \& Nunes (2018) consideram que a reforma psiquiátrica brasileira estava em expansão e conseguiu avanços tal como um maior destino de recursos para atenção psicossocial e fechamento de leitos psiquiátricos. Esta, porém, sofre com as mudanças 
na política de saúde mental desde o final de 2017, a qual prevê recursos para instituiçóes fechadas até então não componentes da Rede de Atenção Psicossocial (tal como comunidades terapêuticas e hospitais psiquiátricos). Conhecer de que modo os programas de residência têm operado, é importante para a identificação se estão de fato agindo para a consolidação do SUS e ampliação do cuidado em saúde mental.

Destacamos também que os programas apresentaram a educação permanente como necessária para seus processos de formação, favorecendo a reflexão crítica dos profissionais. Além disso, a interprofissionalidade também esteve presente em grande parte das apresentaçôes dos objetivos dos programas de residência em saúde mental, em consonância com o que é esperado das RMS.

A educação permanente pode ser estratégica para a educação e trabalho interprofissional em saúde, mas, para isso, é necessário que sua intencionalidade esteja voltada para o fortalecimento da colaboração. Para Gigante \& Campos (2016), ela é fortalecida a partir da pactuação das estratégias construídas, de modo a envolver diferentes atores tanto do setor da saúde (trabalhadores, usuários e gestores) quanto da educação (instituições de ensino, gestores, estudantes dos cursos da área da saúde). No campo da saúde mental, percebemos que a interprofissionalidade é algo presente em sua história, no desenvolvimento de práticas comuns dos trabalhadores da saúde mental. Nesse sentido, é importante que os programas de RMS fortaleçam essas práticas.

Para a terapia ocupacional, a interprofissionalidade infere uma função política a partir da ampliação de outros profissionais sobre o nosso núcleo profissional, algo que historicamente está presente na luta por identidade que a terapia ocupacional vem tentando afirmar e buscar. Constantinidis \& Cunha (2016) apontam que

O fato de a falta de fronteiras que demarcam nosso território de atuação na saúde mental ser constituinte de nossa identidade faz com que estejamos abertos à imanência deste campo e possamos colaborar para que a produção coletiva ganhe potência (Constantinidis \& Cunha, 2016, p. 55).

Atualmente, 127 programas de residência multiprofissional incluem a terapia ocupacional $^{3}$. Os programas em Saúde Mental representam 25\% desses programas. A Figura 3 mostra a distribuição nas diferentes áreas/campos de atuação dos programas de residência multiprofissional com vagas para terapia ocupacional.

$\mathrm{Na}$ categoria outros, estão os programas de residência relacionados com as seguintes áreas de concentração: Urgência/Emergência/Trauma; Atenção Cardiopulmonar (3,9\% cada); Gestão (3,1\%); Terapia Intensiva (2,4\%); Saúde do adulto e do idoso; Hospitalar; Cuidados Paliativos (1,6\%); Clínica Médica; Paciente Crítico; Infectologia; Práticas Integrativas e Complementares; Atenção Integral à Saúde; Saúde do Trabalhador e Saúde da População do Campo contam com um único programa, o que representa $0,8 \%$ do total/cada.

Com relação à oferta de programas de residência para terapeutas ocupacionais, verificamos que a Saúde Mental representa a maior oferta (25\%), seguida da Saúde da Família/Atençáo Primária (12\%).

\footnotetext{
${ }^{3}$ De acordo com levantamento realizado pela Executiva Nacional de Estudantes de Terapia Ocupacional (ExNETO, 2021).
} 


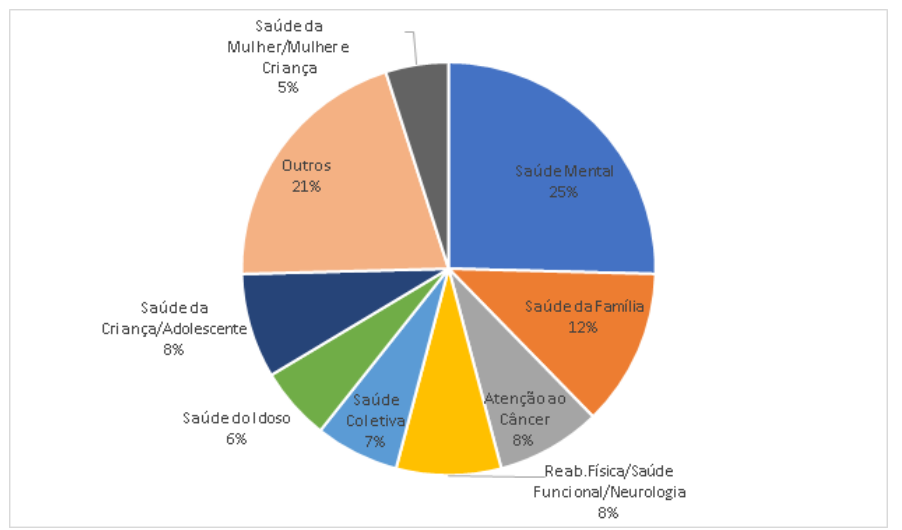

Figura 3. Divisão por área/campo de atuação dos Programas de Residência Multiprofissional que ofertam vagas para a Terapia Ocupacional (2020-2021). Fonte: Elaboraçáo própria (2021).

Para Camargos et al. (2017), a inserção do terapeuta ocupacional no SUS tem sido viabilizada pela implementação dos diferentes programas e políticas e, atualmente, tem o reconhecimento de ser uma categoria profissional potencial nos serviços da Rede de Atenção Psicossocial, Atenção Primária à Saúde e Rede de Urgência e Emergência. Ambas as redes de serviços estão presentes nos cenários de prática que identificamos nos programas de residência em saúde mental com a inserção do terapeuta ocupacional.

Ao considerarmos apenas os programas de residência de saúde mental que incluem o terapeuta ocupacional, São Paulo é o estado que concentra o maior número de programas (8), seguido do Rio Grande do Sul (6).

O Rio Grande do Sul, por sua vez, é o estado com maior número de programas de residência em saúde mental (10 programas no total e 6 deles com terapeutas ocupacionais). Dentre as RMS que ofertam vagas para a terapia ocupacional no estado, a saúde mental representa 38,9\% dos programas, proporção maior do que o encontrado no cenário nacional (de 25\%).

Embora haja um destaque para o número de programas com inserção de terapeutas ocupacionais no Rio Grande do Sul, uma pesquisa realizada pela Câmara Técnica de Saúde Mental do CREFITO-5 em 2018 revelou uma escassez de terapeutas ocupacionais contratados para atuação nos seus serviços públicos de saúde e concentração na capital do estado (Conselho Regional de Fisioterapia e Terapia Ocupacional, 2018).

A insuficiência ou ausência de terapeutas ocupacionais contratados para atuação nos cenários de prática onde se inserem residentes foi observada também por Medina (2016), a qual realizou estudo com três programas de residência multiprofissional (na atenção hospitalar e na atenção básica). Com isso, foram frequentes os relatos de desconhecimento da atuaçáo profissional do terapeuta ocupacional tanto pelos usuários dos serviços quanto de outros profissionais.

Além do desconhecimento acerca da profissão, é importante destacar que essa insuficiência de profissionais na rede de saúde reflete o sucateamento dos serviços públicos e a utilização da residência como máo de obra dos serviços, o que prejudica seu aspecto formativo. Esse aspecto é destacado por Silva (2018), ao considerar que o fato de ser caracterizado por ensino e serviço a torna 
[...] de um lado, uma possibilidade de formação interdisciplinar conectada com o cotidiano concreto das necessidades de saúde e, de outro, táo vulnerável à sua apreensão como trabalho precário. É justamente por sua característica central que os parâmetros postos na regulamentação dessa formação são indicativos dos sentidos que a RMS vem assumindo no SUS (Silva, 2018, p. 207).

Mais especificamente no campo da saúde mental, já apontamos o processo desmontagem e desfinanciamento que tem sido construído ao longo da história da reforma psiquiátrica brasileira (Amarante \& Nunes, 2018). Tais aspectos impactam na inserção dos profissionais no campo da saúde mental e na sua formação qualificada, na qual a terapia ocupacional se insere.

Do ponto de vista da especificidade da terapia ocupacional nestas residências, o que foi localizado nos editais foram as referências bibliográficas indicadas, as quais estiveram presentes em 13 dos 31 programas. Ao olharmos apenas para as produçóes que articulavam a terapia ocupacional e a saúde mental, foram identificadas 54 diferentes indicaçóes de referências bibliográficas, sendo a maioria de autoras da região sudeste.

A partir da leitura de suas palavra-chave, verificou-se nas produçóes uma pluralidade tanto no que diz respeito aos conceitos adotados, tipos de intervençóes, público-alvo e locais de atuação. Além de "terapia ocupacional”(34) e "saúde mental"(36), as palavraschave mais presentes nas produçóes, e a proporção que apareceram, são: "avaliação" e "serviços de saúde mental” (5); "reabilitação psicossocial”, "políticas públicas”, "terapia ocupacional/tendências"; "drogas" e "criança" (4); "desinstitucionalização", "institucionalização" e "inclusão social" (3); "atividades humanas"; "atividades cotidianas", "Sistema Único de Saúde”, "pessoas em situaçáo de rua”, "adolescência”, "encaminhamento e consulta", "métodos", "grupo"; "economia solidária, "cooperativismo", "trabalho", "participação social”, "reabilitação", "centros de reabilitação", "atenção à saúde", "transtornos mentais"; "transtornos relacionados ao uso de substâncias", "terapia não dirigida” e "crise"(2). Já "cotidiano", "reforma psiquiátrica" e "atenção psicossocial" apareceram apenas uma vez, assim como outras 42 palavras.

Para Leão \& Salles (2016), o conceito de cotidiano, pela sua abrangência e articulação com conceitos utilizados na clínica ampliada em reabilitação psicossocial, favorece a fundamentação da clínica de terapeutas ocupacionais no diálogo com outros profissionais da saúde mental, favorecendo o trabalho interdisciplinar.

Embora tenham sido localizados apenas três projetos políticos pedagógicos, uma breve análise do que estes trazem nos permitiu identificar que abordam suas propostas pautando que elas estejam em concordância com as políticas públicas de saúde e de saúde mental.

Outro aspecto abordado é a educação interprofissional como princípio norteador em suas diretrizes pedagógicas. Abordaram que a organização curricular prioriza metodologias de ensino-aprendizagem com foco na interação, proporcionando discussóes e vivências conjuntas das diferentes profissóes envolvidas no cuidado em saúde.

Visando ao desenvolvimento do trabalho em equipe, apontam o desenvolvimento de competências comuns a todos os profissionais de saúde, competências específicas de cada profissão e o desenvolvimento de competências colaborativas, o que está em consonância 
com o proposto por Barr (1998) ao definir as competências para a educação interprofissional.

As competências colaborativas podem ser compreendidas em seis pilares: a comunicação interprofissional; a atenção centrada no indivíduo, família e comunidade; a clareza dos papéis profissionais; o funcionamento da equipe; a liderança colaborativa; e a resolução de conflitos (Canadian Interprofessional Health Collaborative, 2010). Essas competências propostas têm como objetivo nortear as práticas e a formação para o trabalho em equipe interprofissional.

No perfil de competências específico da terapia ocupacional, foi abordada a realização de intervençôes nos territórios, açôes intersetoriais, de forma a contribuir para a modificação dos contextos que geram a exclusão e a invalidação e para o aumento do poder de contratualidade dos usuários. A reabilitação psicossocial também foi um conceito presente, destacando a importância da atuação do terapeuta ocupacional para ampliação das experiências dos usuários nos territórios de existência, ressignificando seu lugar social.

No que tange às especificidades do terapeuta ocupacional na saúde mental, Almeida $\&$ Trevisan (2011) acreditam que

[...] todas as açóes interdisciplinares propostas pelos serviços substitutivos estão de acordo com os pressupostos teóricos que sustentam a profissão; e, ao terapeuta ocupacional, cabe o desafio de demonstrar competência no cotidiano do trabalho em equipe, pontuando o quanto sua formação e seu conhecimento sobre a atividade humana podem contribuir para alcançar os objetivos da Reabilitação Psicossocial (Almeida \& Trevisan, 2011, p. 304).

Nesse sentido, os programas de residência multiprofissionais em saúde mental tornam-se espaços potentes para a formação de terapeutas ocupacionais que, com suas especificidades podem fortalecer a interprofissionalidade e o cuidado em saúde mental, reafirmando seu compromisso histórico com a RP e o Movimento da Luta Antimanicomial.

\section{Consideraçóes Finais}

Identificamos que há nos programas de residência multiprofissional em saúde mental, no que se refere à sua distribuição geográfica, uma manutenção de tendência de centralização nas regióes Sul e Sudeste; porém, verifica-se uma expansão para a região Nordeste. Percebemos ainda que há um processo de interiorização, conforme preconizado pelos programas de RMS, embora insuficiente.

Com relação às vagas disponibilizadas para a terapia ocupacional, percebemos que, embora haja uma insuficiência de vagas, quando comparado a outras profissóes, o campo da saúde mental representa a maior oferta de vagas em residências para terapeutas ocupacionais. Tal fator pode estar relacionado à contribuição e compromisso histórico da terapia ocupacional com o campo da saúde mental e alinhado ao movimento da luta antimanicomial e reforma psiquiátrica.

Consideramos que o estudo favoreceu o conhecimento acerca da inserção da terapia ocupacional nos programas de residência multiprofissional, a partir do mapeamento 
realizado sobre sua distribuição regional e oferta de vagas. Conhecer o âmbito de formação e inserção profissional da terapia ocupacional é importante para a sua qualificação. Observamos, porém, a necessidade de aprofundar o conhecimento desses programas, tal como investigar se há ociosidade de vagas, identificar as propostas pedagógicas dos programas tanto no campo da saúde mental como no núcleo profissional e sua articulação com as políticas públicas, as quais são atravessadas pela conjuntura de contrarreformas, e que impactam na consolidação das residências multiprofissionais.

Apontamos como limitação do estudo a realização de buscas de editais de seleção apenas das turmas atuais de residentes (ingresso 2020 e 2021), não sendo possível acompanhar a ampliação e redução de vagas de modo transversal, assim como as dificuldades relacionadas às buscas dos dados, as inconsistências em sua lista oficial e o pequeno número de projetos políticos pedagógicos, que permitiram apenas uma breve aproximação das especificidades. Além disso, a análise documental não permite compreender de que modo esses processos formativos estão acontecendo qualitativamente, tanto na cotidianidade dos espaços acadêmicos quanto dos cenários de práticas.

A formação interprofissional pode favorecer uma ampliação da perspectiva de outros profissionais acerca da terapia ocupacional e suas especificidades na dinâmica do trabalho compartilhado em equipe.

\section{Referências}

Almeida, D. T., \& Trevisan, E. R. (2011). Estratégias de intervenção da Terapia Ocupacional em consonância com as transformações da assistência em saúde mental no Brasil. Interface: Comunicação, Saúde, Educação, 15(36), 299307. Recuperado em 2 de fevereiro de 2020, de https://www.scielosp.org/pdf/icse/2011.v15n36/299-308/pt

Amarante, P. (2007). Saúde Mental \& Atenção Psicossocial. Rio de Janeiro: Editora Fiocruz.

Amarante, P., \& Nunes, M. O. (2018). A reforma psiquiátrica no SUS e a luta por uma sociedade sem manicômios. Ciência \& Saúde Coletiva, 23(6), 2067-2074. Recuperado em 15 de dezembro de 2020, de https://www.scielo.br/pdf/csc/v23n6/1413-8123-csc-23-06-2067.pdf

Barr, H. (1998). Competent to collaborate: towards a competency-based model for interprofessional education. Journal of Interprofessional Care, 12(2), 181-188. Recuperado em 10 de abril de 2021, de https://neipc.ufes.br/sites/neipc.ufes.br/files/field/anexo/competent_to_collaborate.pdf

Brasil. (2006). Residência multiprofissional em saúde: experiências, avanços e desafios. Brasília: Ministério da Saúde.

Brasil. (2011, 23 de dezembro). Portaria no 3088/GM/MS, de 23 de dezembro de 2011. Institui a Rede de Atençáo Psicossocial para pessoas com sofrimento ou transtorno mental e com necessidades decorrentes do uso de crack, álcool e outras drogas, no âmbito do Sistema Único de Saúde (SUS). Diário Oficial [da] República Federativa do Brasil, Brasília.

Brasil. (2014, 23 de dezembro). Portaria Interministerial no 16, de 22 de dezembro de 2014. Altera a Portaria Interministerial no 1.077/MEC/MS, de 12 de novembro de 2009, a Portaria Interministerial no 1.320/MEC/MS, de 11 de novembro de 2010 e revoga a Portaria Interministerial no 1.224/MEC/MS, de 3 de outubro de 2012, para atualizar o processo de designação dos membros da Comissão Nacional de Residência Multiprofissional em Saúde (CNRMS) e para incluir áreas profissionais para a realizaçáo de Programas de Residência Multiprofissional e em Área Profissional da Saúde. Diário Oficial [da] República Federativa do Brasil, Brasília, seção 1, p. 21. Recuperado em 10 de janeiro de 2021, de https://portalarquivos2.saude.gov.br/images/pdf/2015/janeiro/08/portariaMEC-16-22122014.pdf 
Camargos, M. A., Ferreira, M. V. B., Maxta, B. S. B., \& Tomasi, A. R. P. (2017). A inserção dos terapeutas ocupacionais na rede de atenção à saúde do SUS em Minas Gerais entre os anos de 2005 e 2015. Cadernos Brasileiros de Terapia Ocupacional, 25(2), 363-372. Recuperado em 12 de janeiro de 2021, de www.cadernosdeterapiaocupacional.ufscar.br/index.php/cadernos/article/view/1732

Canadian Interprofessional Health Collaborative - CIHC. (2010). A National Interprofessional Competency Framework. Vancouver: CIHC.

Ceccim, R. B., \& Feuerwerker, L. C. M. (2004). O quadrilátero da formação para a área da saúde: ensino, gestão, atenção e controle social. Physis, 14(1), 41-65. Recuperado em 12 de dezembro de 2020, de https://www.scielo.br/pdf/physis/v14n1/v14n1a04.pdf

Coelho, T. A. D., Nunes, M. O., \& Barreto, S. M. G. (2017). Residência em saúde mental: educando trabalhadores para a atenção psicossocial. Salvador: EDUFBA.

Conselho Regional de Fisioterapia e Terapia Ocupacional - CREFITO-5. (2018). Informativo das Câmaras Técnicas de Terapia Ocupacional. Recuperado em 3 de maio de 2021, de https:/crefito5.org/images/uploads/publications/noAdQXFFDnhFpbh0iaoCPNkQrEoEU8U2P6wQcacy.pdf.

Constantinidis, T. C., \& Cunha, A. C. (2016). Desinstitucionalizando conceitos: a terapia ocupacional em busca de um (novo) lugar no cenário da saúde mental. In T. S. Matsukura \& M. M. Salles (Eds.), Cotidiano, atividade humana e ocupaçâo: Perspectivas da terapia ocupacional no campo da saúde mental (pp. 37-59). São Carlos: EDUFSCar.

Emerick, B. F., \& Onocko-Campos, R. T. (2016). Formação de Trabalhadores para a saúde mental: a experiência da Residência Multiprofissional no Projeto "Percursos Formativos em Desinstitucionalização” In: S. D. Rosa (Org.), Formação profissional em saúde mental: experiências, desafios e contribuiçôes da residência multiprofissional em saúde (pp. 93-107). Curitiba: CRV.

Executiva Nacional de Estudantes de Terapia Ocupacional - ExNETO. (2021). Divulgação de residências de terapia ocupacional no pais. Recuperado em 28 de janeiro de 2021, de https://drive.google.com/folderview?id=1C2Tx_J0AtmO4TjkA2zizDVbO5Mwa9AA-.

Garcia Junior, C. A. S., \& Yasui, S. (2018). Reflexôes sobre a formação para o SUS e sua articulação com a pesquisa e a in(ter)venção nos cenários das práticas e dos serviços. Interação em Psicologia, 22(3), 158166. Recuperado em 5 de janeiro de 2021, de https://revistas.ufpr.br/psicologia/article/view/56076

Gigante, R. L., \& Campos, G. W. S. (2016). Política de formação e educação permanente em saúde no brasil: bases legais e referências teóricas. Trabalho, Educação e Saúde, 14(3), 747-763. http://dx.doi.org/10.1590/1981-7746-sip00124.

Gil, A. C. (2018). Como elaborar projetos de pesquisa. São Paulo: Atlas.

Leão, A., \& Salles, M. M. S. (2016). Cotidiano, Reabilitação Psicossocial e Território: reflexões no campo da terapia ocupacional. In T. S. Matsukura \& M. M. Salles (Eds.), Cotidiano, atividade humana e ocupação: perspectivas da terapia ocupacional no campo da saúde mental (pp. 37-59). São Carlos: EDUFSCar.

Lins, S. R. A., \& Matsukura, T. S. (2015a). Desafios e perspectivas da formação do terapeuta ocupacional no campo da saúde mental. Revista de Terapia Ocupacional da Universidade de São Paulo, 26(3), $317-$ 325. Recuperado em 7 de janeiro de 2021, de ww.revistas.usp.br/rto/article/view/100867

Lins, S. R. A., \& Matsukura, T. S. (2015b). A formação graduada do terapeuta ocupacional no campo da saúde mental: a perspectiva de discentes e egressos. Cadernos de Terapia Ocupacional da UFSCar, 23(4), 689-699. Recuperado em 15 de janeiro de 2021, de www.cadernosdeterapiaocupacional.ufscar.br/index.php/cadernos/article/view/1192

Lussi, I. A. O., Ferigato, S. H., Gozzi, A. P. N. F., Fernandes, D. S. A., Morato, G. G., Cid, M. F. B., Furlan, P. G., Marcolino, T. Q., \& Matsukura, T. S. (2019). Saúde mental em pauta: afirmação do cuidado em liberdade e resistência aos retrocessos. Cadernos Brasileiros de Terapia Ocupacional, 27(1), 1-3. Recuperado em 18 de dezembro de 2020, de https://www.scielo.br/scielo.php?script=sci_arttext\&pid=S2526$89102019000100001 \& \operatorname{lng}=\mathrm{pt} \& \mathrm{nrm}=\mathrm{iso} \& \mathrm{t} \operatorname{lng}=\mathrm{pt}$

Medina, A. G. (2016). A terapia ocupacional e a educação para a interprofissionalidade em residências multiprofissionais em saúde (Dissertação de mestrado). Universidade de São Paulo, São Paulo. 
Onocko-Campos, R. T., Emerick, B. F., \& Ricci, E. C. (2019). Residência Multiprofissional em Saúde Mental: suporte teórico para o percurso formativo. Interface: Comunicação, Saúde, Educação, 23, 1-13. Recuperado em 12 de janeiro de 2021, de https://www.scielo.br/pdf/icse/v23/1807-5762-icse-23-e170813.pdf

Organização Mundial da Saúde - OMS. (2010). Marco para a ação em educação interprofissional e prática colaborativa. Recuperado em 2 de maio de 2021, de https:/www.educacioninterprofesional.org/sites/default/files/fulltext/2018/pub_oms_marco_acao_eip.pdf.

Palm, R. C. M., Nimtz, M. A., \& Mantovani, M. F. (2015). Formação em saúde mental nos cursos de Terapia Ocupacional da região sul do Brasil. Cogitare Enfermagem, 20(2), 289-298. Recuperado em 08 de dezembro de 2020, de https://revistas.ufpr.br/cogitare/article/view/41423

Peduzzi, M., Agreli, H. L. F., Silva, J. A. M., \& Silva, H. S. (2020). Trabalho em equipe: uma revisita ao conceito e a seus desdobramentos no trabalho interprofissional. Trabalho, Educação e Saúde, 18, 1 20. Recuperado em 11 de dezembro de 2020, de https:/www.scielo.br/scielo.php?script= sci_arttext \&pid=S1981-77462020000400401 \& tlng=pt

Poellnitz, J. C. V., \& Silva, C. R. (2019). Sobre a linguagem: sentidos para uso de termos e conceitos. In C. R. Silva (Org.), Atividades Humanas \& Terapia Ocupacional: Saber-fazer, cultura, politica e outras resistências (pp. 80-96). São Paulo: Hucitec.

Sarmento, L. F., França, T., Medeiros, K. R., Santos, M. R., \& Ney, M. S. (2017). A distribuição regional da oferta de formação na modalidade Residência Multiprofissional em Saúde. Saúde Debate, 41(113), 415424. Recuperado em 7 de janeiro de 2021, de https://www.scielo.br/pdf/sdeb/v41n113/0103-1104-sdeb41-113-0415.pdf

Sá-Silva, J. R., Almeida, C. D., \& Gundani, J. F. (2009). Pesquisa documental: pistas teóricas e metodológicas. Revista Brasileira de História \& Ciências Sociais, 1(1), 1-15. Recuperado em 15 de abril de 2021, de https://periodicos.furg.br/rbhcs/article/view/10351

Silva, L. B. (2018). Residência Multiprofissional em Saúde no Brasil: alguns aspectos da trajetória histórica Revista Katálysis, 21(1), 200-209. Recuperado em 12 de janeiro de 2021, de https:/www.scielo.br/pdf/rk/v21n1/1414-4980-rk-21-01-00200.pdf

Torres, R. B. S., Barreto, I. C. H. C., Freitas, R. W. J. F., \& Evangelista, A. L. P. (2019). Estado da arte das residências integradas, multiprofissionais e em área profissional da Saúde. Interface: Comunicação, Saúde, Educação, 23, 1-16. Recuperado em 12 de janeiro de 2021, de htps://www.scielo.br/pdf/icse/v23/18075762-icse-23-e170691.pdf

Yasui, S. (2010). Rupturas \& encontros: desafios da reforma psiquiátrica brasileira. Rio de Janeiro: Editora Fiocruz.

\section{Contribuiçáo dos Autores}

Aline Silva de Moura realizou a coleta, organizaçáo e análise dos dados, elaboração e redação do texto. Éllen Cristina Ricci contribuiu na discussão dos dados, redação e revisão do texto. Sabrina Helena Ferigato realizou a orientação do trabalho, organização e análise dos dados, redação e revisão do texto. Todas as autoras aprovaram a versão final do texto.

\section{Autor para correspondência}

Aline Silva de Moura

e-mail: alinesmoura86@gmail.com

\section{Editor de seçáo}

Prof. Dr. Milton Carlos Mariotti 